\title{
Innovationsgesellschaft heute
}


Werner Rammert · Arnold Windeler Hubert Knoblauch - Michael Hutter (Hrsg.)

\section{Innovations- gesellschaft heute Perspektiven, Felder und Fälle}

Springer VS 
Herausgeber

Werner Rammert

Berlin, Deutschland

Arnold Windeler

Berlin, Deutschland

\author{
Hubert Knoblauch
}

Berlin, Deutschland

Michael Hutter

Berlin, Deutschland

ISBN 978-3-658-10873-1

ISBN 978-3-658-10874-8 (eBook)

DOI 10.1007/978-3-658-10874-8

Die Deutsche Nationalbibliothek verzeichnet diese Publikation in der Deutschen Nationalbibliografie; detaillierte bibliografische Daten sind im Internet über http://dnb.d-nb.de abrufbar.

\section{Springer VS}

(C) Springer Fachmedien Wiesbaden 2016

Das Werk einschließlich aller seiner Teile ist urheberrechtlich geschützt. Jede Verwertung, die nicht ausdrücklich vom Urheberrechtsgesetz zugelassen ist, bedarf der vorherigen Zustimmung des Verlags. Das gilt insbesondere für Vervielfältigungen, Bearbeitungen, Übersetzungen, Mikroverfilmungen und die Einspeicherung und Verarbeitung in elektronischen Systemen.

Die Wiedergabe von Gebrauchsnamen, Handelsnamen, Warenbezeichnungen usw. in diesem Werk berechtigt auch ohne besondere Kennzeichnung nicht zu der Annahme, dass solche Namen im Sinne der Warenzeichen- und Markenschutz-Gesetzgebung als frei zu betrachten wären und daher von jedermann benutzt werden dürften.

Der Verlag, die Autoren und die Herausgeber gehen davon aus, dass die Angaben und Informationen in diesem Werk zum Zeitpunkt der Veröffentlichung vollständig und korrekt sind. Weder der Verlag noch die Autoren oder die Herausgeber übernehmen, ausdrücklich oder implizit, Gewähr für den Inhalt des Werkes, etwaige Fehler oder Äußerungen.

Lektorat: Cori Antonia Mackrodt, Kerstin Hoffmann

Gedruckt auf säurefreiem und chlorfrei gebleichtem Papier

Springer VS ist Teil von Springer Nature

Die eingetragene Gesellschaft ist Springer Fachmedien Wiesbaden GmbH 


\section{Inhaltsverzeichnis}

\section{Einleitung}

Die Ausweitung der Innovationszone $\ldots \ldots \ldots \ldots \ldots \ldots \ldots \ldots \ldots$

Werner Rammert, Arnold Windeler, Hubert Knoblauch

und Michael Hutter

Innovationsgesellschaft heute

Die reflexive Herstellung des Neuen

Michael Hutter, Hubert Knoblauch, Werner Rammert und Arnold Windeler

\section{Teil I Sozial- und gesellschaftstheoretische Perspektiven}

Fragmentale Differenzierung und die Praxis der Innovation . . . . . . . 39

Wie immer mehr Innovationsfelder entstehen

Jan-Hendrik Passoth und Werner Rammert

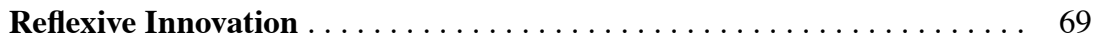

Zur Innovation in der radikalisierten Moderne

Arnold Windeler

Kommunikatives Handeln, das Neue und die Innovationsgesellschaft . . 111 Hubert Knoblauch

Das Kreativitätsdispositiv und die sozialen Regime des Neuen . . . . . . . 133

Andreas Reckwitz 


\section{Teil II Zwischen Wirtschaft und Kultur}

Zur Rolle des Neuen in der Erlebniswirtschaft . . . . . . . . . . . 157

Michael Hutter

Strategisches Marketing in der Innovationsgesellschaft $\ldots \ldots \ldots \ldots .175$

Ein Bezugsrahmen

Franz Liebl

Innovation mit Hilfe der Vielen

Crowdsourcing im Innovationsprozess

Arnold Picot und Stefan Hopf

Das Berliner Innovationspanel

Entstehungsgeschichte, erste Ergebnisse und Ausblick

Knut Blind

Innovation: In Ketten tanzen.

Günther Ortmann

\section{Teil III Zwischen Politik, Planung und sozialer Bewegung}

Flash Mobs als Innovation

Über eine neue Sozialform technisch vermittelter Versammlung

Paul Gebelein, Martina Löw und Thomas Paul

Wie kommt Neuartiges in die räumliche Planung?.

Konzeptionierung von Innovationen in der Planung und Forschungsstrategien

Gabriela Christmann, Oliver Ibert, Johann Jessen

und Uwe-Jens Walther

Energiewende

Pfadbruch oder Manifestierung des Ausgangspfades?

Johann Köppel

Governance-Innovationen

Epistemische und politische Reflexivitäten in der Herstellung von Citizen Panels Jan-Peter $\mathrm{Voß}$ 


\section{Teil IV Zwischen Wissenschaft und Innovationspolitik}

Epistemische Innovation .......................... 355

Zur Entstehung des Neuen in der Wissenschaft aus Sicht der Science Studies Martina Merz

Organisationale Innovation am Beispiel der Projektifizierung der Wissenschaft

Eine figurationssoziologische Perspektive auf Entstehung,

Verbreitung und Wirkungen

Nina Baur, Cristina Besio und Maria Norkus

Soziale Innovationen.

Kontrollverluste und Steuerungsversprechen sozialen Wandels

Cornelius Schubert

Pläne und die Zukunft

Das Unvorhersagbare gestalten

Elena Esposito 